\title{
artigo
}

\section{Evolução dos casos de COVID-19 de dezembro de 2019 a outubro de 2020 no Maranhão}

\author{
Evolution of COVID-19 cases from december 2019 to october 2020 in Maranhão \\ Evolucón de los casos COVID-19 desde diciembre de 2019 a octubre de 2020 en Maranhão
}

\begin{abstract}
RESUMO
No final de dezembro de 2019, em Wuhan, uma nova variedade de Corona vírus foi identificada, sendo declarada como uma emergência de saúde pública pela Organização Mundial de Saúde. OBJETIVO: Analisar a evolução epidemiológica do COVID-19 no estado do Maranhão. MÉTODOS: Trata-se de um estudo epidemiológico descritivo e retrospectivo, com abordagem quantitativa. RESULTADOS: O Maranhão representa $1,7 \%$ dos casos no Brasil, figurando na $5^{\circ}$ colocação na região Nordeste. Em termos de número de óbitos por Síndrome Respiratória Aguda Grave, o mesmo estado contribuiu com 2,1\% dos casos brasileiros, sendo sua predominância em indivíduos do sexo feminino (56\%) e na faixa etária entre 30 a 49 anos (37,5\%). CONCLUSÃO: Ressalta-se a importância de promover mais estudos sobre a doença e investir em estratégias para o controle do coronavírus, pois o surto do COVID-19 é recente e sua duração é incerta.
\end{abstract}

DESCRITORES: Coronavirus; Saúde Pública; Epidemiologia descritiva.

\section{ABSTRACT}

At the end of December 2019, in Wuhan, a new variety of Corona virus was identified, being declared as a public health emergency by the World Health Organization. OBJECTIVE: To analyze the epidemiological evolution of COVID-19 in the state of Maranhão. METHODS: This is a descriptive and retrospective epidemiological study, with a quantitative approach. RESULTS: Maranhão represents $1.7 \%$ of the cases in Brazil, ranking 5th in the Northeast region. In terms of number of deaths due to Severe Acute Respiratory Syndrome, the same state contributed with $2.1 \%$ of Brazilian cases, with its predominance in females $(56 \%)$ and in the age group between 30 and 49 years (37.5\%). CONCLUSION: The importance of promoting further studies on the disease and investing in strategies to control the coronavirus is emphasized, since the outbreak of COVID-19 is recent and its duration is uncertain.

DESCRIPTORS: Coronavirus; Public Health; Descriptive epidemiology.

\section{RESUMEN}

A fines de diciembre de 2019, en Wuhan, se identificó una nueva variedad del virus Corona, declarada emergencia de salud pública por la Organización Mundial de la Salud. OBJETIVO: Analizar la evolución epidemiológica del COVID-19 en el estado de Maranhão. MÉTODOS: Se trata de un estudio epidemiológico descriptivo, retrospectivo, con enfoque cuantitativo. RESULTADOS: Maranhão representa el 1,7\% de los dos casos en Brasil, ocupando el quinto lugar en la región Nordeste. En cuanto al número de defunciones por Síndrome Respiratorio Agudo Severo, o el mismo estado, dos casos brasileños aportaron el 2,1\%, con predominio de mujeres (56\%) y un grupo de 30 a 49 años (37, 5\%). CONCLUSIÓN: Se enfatiza la importancia de impulsar más estudios sobre la enfermedad e invertir en estrategias para controlar el coronavirus, ya que el brote de COVID-19 es reciente y su duración es incierta.

DESCRIPTORES: Coronavirus; Salud Pública; Epidemiología Descriptiva.

RECEBIDO EM: 15/12/2020 APROVADO EM: 07/01/2021

\section{Bárbara dos Santos Bezerra}

Acadêmica do curso de Enfermagem na Universidade Estadual do Maranhão (UEMA).

ORCID: 0000-0001-5177-1591

\section{Sandra Regina Matos da Silva}

Enfermeira; docente substituto (UEMA/CESSIN); mestranda em saúde e ambiente pela Universidade Federal do Maranhão, especialista em Saúde da família.

ORCID: 0000-0002-3771-4088 


\section{Thaynara Pinheiro Araújo}

Acadêmica do curso de Enfermagem na Universidade Estadual do Maranhão (UEMA).

ORCID: 0000-0002-5256-5738

\section{INTRODUÇÃO}

$\mathbf{N}$ o final de dezembro de 2019 , em Wuhan, uma cidade na china surgiu uma nova variedade do Corona vírus, sendo declarado com uma emergência de saúde pública pela Organização Mundial de Saúde (OMS). Esse vírus é denominado SARS-CoV2 e produz uma doença intitulada como COVID-19, que pode variar desde uma simples gripe até casos mais graves (com grande prejuízo respiratório), colocando a vida dos acometidos em risco ${ }^{1}$.

No Brasil, no dia 3 de fevereiro de 2020, foi declarada por meio da portaria nº 188 do Ministério da saúde, emergência em saúde pública de importância nacional, devido a ocorrência da infecção humana pelo novo coronavírus, isso com o intuito de que o país desenvolvesse medidas de prevenção/controle para enfrentar essa nova pandemia. De acordo com o Ministério da saúde (MS), o primeiro caso de infecção no Brasil pelo novo coronavírus, foi no dia 26 de fevereiro em São Paulo, e a partir desse novo caso todo o país ficou em alerta ${ }^{2}$.

Uma característica do SARS-CoV2 que contribuiu para que ele se tornasse um problema mundial, foi a sua forma de transmissão, que ocorre através do contato direto com o próprio vírus, ou seja, as gotículas de espirros ou tosse dos infectados entram em contato com as mucosas (boca, olho e nariz), causando a infecção pelo coronavírus. Ele pode sobreviver fora de um hospedeiro por mais de 72 horas, por isso corrimões e maçanetas são locais onde pode se tornar um potencial ambiente de contaminação, então é importante evitar aglomerações e manter uma distância mínima de 1,5 metros de outras pessoas, para evitar o contato com as gotículas e não contrair o vírus ${ }^{1}$.

Diante do aumento expressivo do COVID-19 em todo o mundo, as pessoas devem tomar medidas preventivas es- pecificas, que consequentemente causam impacto na vida dos seres humanos e na sociedade, pois a restrição do contanto social pode trazer consequências para a saúde mental, além dos trabalhadores estarem impedidos de ir até seu local de trabalho, passando a trabalhar de forma remota correndo o risco de perder seu emprego, sua fonte de renda ${ }^{1}$.

No final de dezembro de 2019, em Wuhan, uma cidade na china surgiu uma nova variedade do Corona vírus, sendo declarado com uma emergência de saúde pública pela Organização Mundial de Saúde (OMS).

Os sintomas da COVID-19 incluem febre alta, tosse persistente e dificuldade de respirar, as complicações desta infecção costumam surgir especialmente em pessoas idosas com idade superior a 60 anos, pois o vírus tem uma agressividade maior em organismos com comorbidades (com doenças pré existentes), especialmente cardíacas e diabéticas, porém, isso não descarta a impossibilidade de óbito em pessoas saudáveis ${ }^{3}$.
Nessa perspectiva este trabalho se faz necessário para entender e responder a pergunta: "Como ocorreu a evolução do Covid-19?”, visando isso a pesquisa se propôs a analisar a evolução epidemiológica do COVID-19, da mesma forma explorando a experiência mundial de medidas de prevenção e controle preconizadas pela Organização Mundial de saúde.

\section{MÉTODOS}

Trata-se de um estudo epidemiológico descritivo, retrospectivo com abordagem quantitativa. A pesquisa aconteceu entre os meses de Setembro a Novembro de 2020 e os dados foram obtidos através dos boletins epidemiológico do Ministério da Saúde e da Secretaria Estadual de Saúde do Maranhão, onde foram analisados os boletins emitidos no intermediário de tempo de 10 meses, de dezembro de 2019 até outubro de 2020.

Os principais países analisados no contexto mundial foram: a China, Estados Unidos, Itália, Espanha, Índia e Brasil, analisando se o COVID-19 evoluiu para valores crescentes ou decrescentes com o número de infectados.

Logo em seguida realizamos uma comparação com o Brasil, região Nordeste e Maranhão. Além disso, confrontamos a síndrome respiratória aguda grave com o COVID-19 e investigamos as variáveis de faixa etária e sexo no maranhão, com a finalidade de verificar a prevalência dos casos de COVID-19. Consideramos três variáveis no contexto nacional, regional e estadual: i. Incidência; ii. Letalidade; e iii. Mortalidade. Utilizamos para consolidação dos resultados o programa Office Excel 2016 tanto para criação das tabelas quanto dos gráficos.

Os critérios de inclusão para a análise dos boletins foram: Boletins completos que estivessem dentro do período de dezembro de 2019 a outubro de 2020, e exclui-se os boletins que apresentavam 


\section{artigo}

Bezarra, B.S.; Matos da Silva, S.R.; Araújo, T.P.

Evolução dos casos de COVID-19 de dezembro de 2019 a outubro de 2020 no Maranhão

apenas informações sobre medidas de prevenção e não apresentavam dados informativos sobre os casos do COVID-19.

O referido trabalho dispensou a avaliação do Comitê de Ética em Pesquisa por se tratar de análise de dados epidemiológicos em domínio público, estando de acordo com as Resoluções no 466/2012 e 510/2016 do Conselho Nacional de Saúde que disciplina as pesquisas realizadas com seres humanos.

\section{RESULTADOS}

Para construção das informações foi 1) onde traçamos a linha do tempo de como ocorreu o surgimento dos casos Covid-19 de forma resumida na China e Brasil.

O primeiro caso apresentando sintomas de coronavírus foi ainda em novembro de 2019, sendo confirmado por um estudo retrospectivo de acordo com os boletins do Ministério da Saúde ${ }^{4}$.

Foi relatado que no final de dezembro de 2019, várias unidades de saúde locais da China, receberam grupos de pacientes com pneumonia de causa desconhecida, possível estruturar um fluxograma (Figura

e estavam ligados a um mercado atacadista de frutos do mar e animais úmidos em Wuhan, província de Hubei, China ${ }^{5,6}$.

Em 23 de Janeiro o Brasil notifica o primeiro caso suspeito para Covid-19, a OMS no dia 30 declara emergência de saúde pública de importância internacional nesse período o vírus já havia atingido 24 países e a China já apresentava 7,7 mil casos e 170 óbitos ${ }^{4,7}$.

No dia 26 de Fevereiro, o Brasil tem o seu $1^{\circ}$ caso confirmado na região Sudeste em São Paulo, um homem de 61 anos que deu entrada no Hospital Israelense Albert Einstein, e o segundo caso confirmado no dia 29, também homem, de acordo com as informações os dois haviam acabado de retornar de uma viagem da Itália ${ }^{4,8}$.

O primeiro óbito notificado no Brasil foi em 17 de Março, até o final do mesmo mês o Brasil já contabilizava 4,579 casos e no mundo até o dia 3 de abril já existiam 972.640 casos confirmados de COVID-19 com 50.325 óbitos. Os Estados Unidos da América era o país com maior número de casos, totalizando 213.600, e a Itália acumulava o maior número de óbitos, $13.9174,7$.

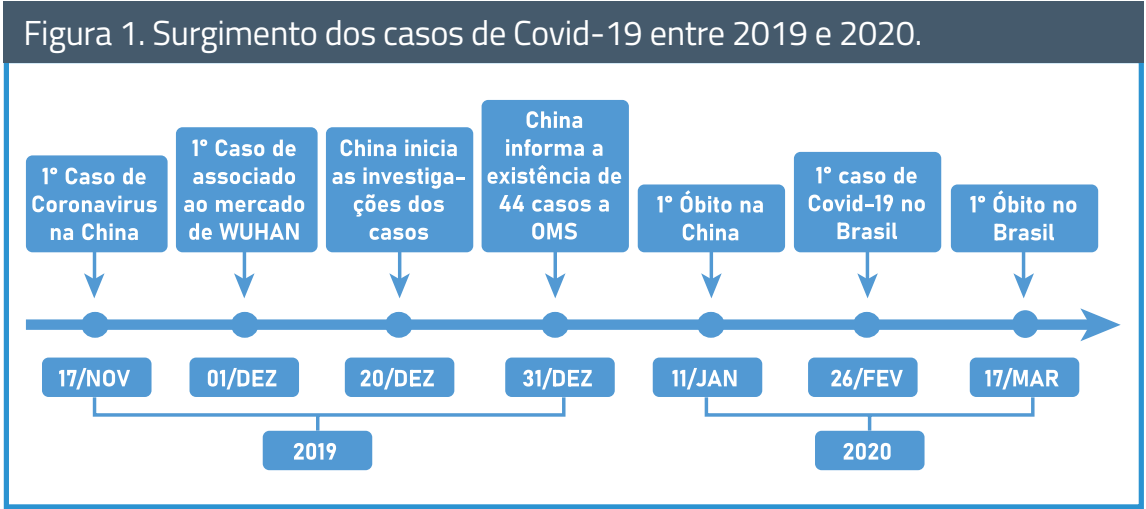

Fonte: Boletim epidemiológico do Ministério da Saúde/Brasil.
No mês de maio o Brasil transcende todos os países e chega a segunda posição no ranking mundial com 347,398 casos confirmados para Covid-19, em Junho a Índia surge no ranking mundial e assume a $3^{\circ}$ posição com 508,953 casos, nesse mesmo período o Brasil já ultrapassava 1 milhão de casos, ainda em $2^{\circ}$ lugar, ficando atrás somente dos Estados Unidos que notificava $2.467,837$ casos .

No Brasil 8,47\% dos casos de COVID-19 estavam associados a Síndrome Respiratória Aguda Grave (SRAG), sendo que os casos notificados no mesmo período para SRAG, 54\% deles eram por conta do Covid-19. A associação da SRAG com o COVID-19 em relação aos óbitos teve um número ainda mais expressivo, pois $10,6 \%$ desses óbitos estavam ligados a SRAG e 70\% dos óbitos notificados por de SRAG foram devidos o COVID-199.

$\mathrm{Na}$ tabela abaixo iremos acompanhar a evolução do COVID-19 na região nordeste, dando ênfase no Maranhão, no período também de abril a outubro de 2020.

Grande parte dos casos no Maranhão, concentravam-se na capital contabilizando $76,1 \%$ dos casos em Abril, maior parte dos óbitos relatados no estado do Maranhão e da região nordeste nesse período, eram idosos do sexo masculino e com comorbidades como diabetes e cardiopatias.

Foi observado que o COVID-19 atingiu $2,4 \%$ da população brasileira e da região nordeste e no Maranhão 2,6\%, um número menor comparado a região $\mathrm{Su}$ deste em específico em São Paulo, que a prevalência de infectados pelo novo coronavírus chegou a $11,1 \%$ da população, ou seja, 1,32 milhões ${ }^{2,10}$.

A taxa de mortalidade e letalidade também foram semelhantes, porém ressaltan-

Tabela 1. Evolução dos casos de Covid-19 da região Nordeste, Maranhão e São Luís.

\begin{tabular}{lccccccc}
\multicolumn{7}{c}{ EVOLUÇÃO DOS CASOS DE COVID-19 NA REGIÃO NORDESTE, MARANHÃO E NA CAPITAL SÃO LUÍS. } \\
LOCAL & ABRIL & MAIO & JUNHO & JULHO & AGOSTO & SETEMBRO & OUTUBRO \\
NORDESTE & 17,531 & 119,801 & 451,076 & 87,1373 & $1.191,103$ & $1.384,855$ & $1.417,339$ \\
MARANHÃO & 3,506 & 35,297 & 80,451 & 120,061 & 15,616 & 173,564 & 185,341 \\
SÃO LUÍS & 2,671 & 9,255 & 13,050 & 16,160 & 18,796 & 21,098 & 23,272 \\
Fonte: Boletim epidemiológico do Ministério da Saúde-Brasil, SES-MA. & & & & &
\end{tabular}


do que há lugares no Brasil em que esses indicativos muitas vezes foram superiores ao do país, como exemplo a taxa de mortalidade na região sudeste $(79,0)$ e no estado do Ceará $(100,8)$ no nordeste $e^{8,11}$.

Foi observado a predominância do sexo feminino com $55,8 \%$ dos casos e a faixa etária entre 30 a 49 anos totalizando $38 \%$ dos casos, no Brasil no início dos boletins epidemiológico, por volta do mês de maio foi realizada uma análise do perfil das pessoas mais afetadas e foi relatado a prevalência do sexo feminino com $52,8 \%$ dos $\operatorname{casos}^{12}$.

No período observado a raça dos casos notificados que se encontram com maior número, foram a parda e a amarela, sendo respetivamente $39,40 \%$ e $20,40 \%$, cerca de $25 \%$ dos casos não tiveram a raça identificada. No Brasil a raça parda e branca que foram identificadas como dominantes, porém 51,3\% não havia informações, pois os dados não haviam sidos preenchidos, dificultando assim informações com maior credibilidade $^{12}$.

\section{DISCUSSÃO}

Apesar de haver grandes esforços em diferentes continentes, ainda não temos consistência dos fatos sobre a doença e continua-se a busca pela vacina como o melhor meio de prevenção contra o vírus.

Nos estudos realizados na China, há relatos que o primeiro paciente que foi admitido no hospital com os sintomas do COVID-19 foi em 12 de dezembro de 2019, sendo assim, há informações não são condizentes, trazendo abertura para hipótese levantada que o vírus realmente já circulava no país oriental antes das notificações a OMS $S^{13,14,15}$.

Durante a análise da evolução do COVID-19 no Brasil, foi possível observar que o vírus potencializou a SRAG, no período de 10 a 16 de maio de 2020 aconteceu o maior número de casos de SRAG e de óbitos, entre 26 de abril e 2 maio de 2020. Nas bibliografias analisas, foram observados que os casos para SRAG em anos anteriores estiveram abaixo dos números que notificamos agora em plena pande- mia, um deles realizado em Pernambuco observou a SRAG entre 2015 a 2019 (pré-pandemia) e agora 2020 (período pandêmico), ou seja, o Covid-19 gerou um aumento, que no geral nos faz perceber que essa pandemia trouxe à tona o quanto é necessário de medidas para controle e de disseminação das doenças respiratórias ${ }^{1,16}$.

Apesar de haver grandes esforços em diferentes

continentes, ainda não temos consistência dos fatos sobre a doença e continua-se a busca pela vacina como o melhor meio de prevenção contra o vírus.

A evolução do vírus na região Nordeste foi rápida e persistente, essa região é a segunda mais afetada do Brasil estando atrás somente da região sudeste de onde surgiu as primeiras notificações e o Maranhão era um dos estados mais atingidos da região, sendo o $6^{\circ}$ estado no ranking nacional de número de casos notificados.

Foi notificado no dia 20 de março o primeiro caso no estado do Maranhão, um idoso, em estado grave e que recentemente havia chegado de São Paulo. No final de Março o Maranhão já fechava o mês com 52 casos e 1 óbito, e durante todo período a evolução do vírus se deu cada vez mais crescente $^{16}$.
Algo relevante a ser destacado, sobre a testagem da população, a maioria dos testes realizados são do tipo molecular, porém no nordeste e no Maranhão é frequente o uso os testes sorológicos, mas a recomendação é a utilização do exame molecar, ou seja, o swab em todos, já que ele identifica o vírus até em pessoas assintomáticas, porém esse tipo de teste necessita de profissionais treinados e um cuidado maior com o material coletado por ter um teor de contaminação bem maior, colocando em risco os profissionais que realizam o teste ${ }^{2}$.

$\mathrm{O}$ que gerou dificuldade nos interiores do estado do Maranhão, onde a maioria dos profissionais não tinha treinamento específico para a realização dos testes e a testagem em massa seria o ideal, já que fornece informações que deverão modificar as ações de saúde coletiva ${ }^{9,12}$.

Houve uma grande diferença na variável raça, entre os casos do contexto nacional comparado aos do estado do Maranhão, um dos motivos para que a raça parda seja a prevalente no estado pode ser devido sua maior incidência na região, que de acordo com o IBGE, o estado do Maranhão possui $66,5 \%$ da população na raça/cor parda.

Em um estudo realizado no Maranhão no período de março a abril também constatou a prevalência da faixa etária de 30 a 49 anos e do sexo feminino, já a mortalidade no sexo masculino e na faixa etária acima de 60 anos, nos últimos dados coletados no SES os óbitos se tornaram prevalentes também no sexo feminino com $61 \%$ dos casos, a faixa etária continuou a mesma ${ }^{1}$.

Este estudo teve suas limitações que estão diretamente ligadas à constante atualização dos dados em processamento, o que demostra como há uma evolução constate dos casos e transmissão da doença. Assim, é importante que estudos epidemiológicos sejam continuados para avaliar a extensão da pandemia, bem como suas mudanças nos cenários epidemiológicos e sociais no Maranhão.

\section{CONCLUSÃO}

Foi possível observar que houve uma 


\section{artigo}

grande progressão no número de infectados, em todas as faixas etárias e na quantidade de óbito. Desse modo, é de grande relevância a continuação dos estudos para compreender o desfecho da doença, pois ainda não se sabe quais os danos podem gerar a longo prazo.

É necessário que o governo implante ainda mais estratégias para o controle do coronavírus, intensificando em medidas preventivas, e investir na realização dos testes para obter conhecimento da quan- tidade real de pessoas afetadas pelo vírus, com o intuito de reduzir a transmissibilidade e ter conhecimento amplo sobre o perfil populacional atingido, pois o surto do COVID-19 é recente, não há muitos estudos e sua duração é incerta.

\section{REFERÊNCIAS}

1. Silva Anderson Walter Costa, et al. Perfil epidemiológico e determinante social do COVID-19 em Macapá, Amapá, Amazônia, Brasil. Revista Científica Multidisciplinar Núcleo do Conhecimento [Internet]. 2020 Abril [cited 2020 Oct 15];4(4) DOI http://200.139.21.55/handle/123456789/660. Available from: http://200.139.21.55/bitstream/123456789/660/1/Artigo_PerfilEpidemiologicoDeterminante.pdf

2. Oliveira Adriana Cristina de, Lucas Thabata Coaglio, Iquiapaza Robert Aldo. 0 que a pandemia da covid-19 tem nos ensinado sobre adoção de medidas de precaução?. Texto \& Contexto-Enfermagem [Internet]. 2020 [cited 2020 Sep 18];29 DOI https:// doi.org/10.1590/1980-265x-tce-2020-0106. Available from: https://www.scielo.br/scielo.php?\%20pid=S0104-07072020000 100201\&script=sci_arttext\&tlng=pt

3. Araújo Agostinho Antônio Cruz. COVID-19: analysis of confirmed cases in Teresina, Piaui, Brazil. Rev Pre Infec e Saúde [Internet]. 2020 [cited 2020 Sep 30];6 DOI https://doi.org/10.26694/ repis.v6i0.10569. Available from: https://revistas.ufpi.br/index. php/nupcis/article/view/10569

4. Ministério da Saúde (Brasil). Boletim Epidemiológico- Centro de Operações de Emergência em Saúde Pública/Doença pelo Coronavírus 2019(COE-COVID19). Boletim 07 emitido em 06/04/2020. [publicação online] 2020 [cited 2020 Oct 25]. Available from: https://coronavirus.saude.gov.br/boletins-epidemiologicos.

5. Ministério da Saúde (Brasil). Boletim Epidemiológico- Centro de Operações de Emergência em Saúde Pública/Doença pelo Coronavírus 2019(COE-COVID19). Boletim 04 emitido em 04/03/2020. [publicação online] 2020 [cited 2020 Oct 25]. Available from: https://coronavirus.saude.gov.br/boletins-epidemiologicos.

6. Wu Zunyou, McGoogan Jennifer M. Characteristics of and important lessons from the coronavirus disease 2019 (COVID-19) outbreak in China: summary of a report of 72314 cases from the Chinese Center for Disease Control and Prevention. Jama [Internet]. 2020 Jan 28 [cited 2020 Oct 15];323(13):1239-1242. DOI 10.1001 / jama.2020.2648. Available from: https://jamanetwork. com/journals/jama/article-abstract/2762130

7. Ministério da Saúde (Brasil). Boletim Epidemiológico- Centro de Operações de Emergência em Saúde Pública/Doença pelo Coronavírus 2019(COE-COVID19). Boletim 01 emitido em 01/01/2020. [publicação online] 2020 [cited 2020 Oct 25]. Available from: https://coronavirus.saude.gov.br/boletins-epidemiologicos.

8. Ministério da Saúde (Brasil). Boletim Epidemiológico- Centro de Operações de Emergência em Saúde Pública/Doença pelo Coronavírus 2019(COE-COVID19). Boletim 09 emitido em 11/04/2020. [publicação online], 2020 [cited 2020 Oct 25]. Available from: https://coronavirus.saude.gov.br/boletins-epidemiologicos.

9. Almeida Joelson dos Santos. Caracterização epidemiológica dos casos Covid-19 no Maranhão: Uma breve análise. Forthcoming [Internet]. 2020 [cited 2020 Sep 18]; DOI https://doi.org/10.1590/ SciELOPreprints.314. Available from: https://preprints.scielo.org/ index.php/scielo/preprint/view/314

10. Ministério da Saúde (Brasil). Boletim Epidemiológico Especial (BEE). Boletim 36 emitido em 17/10/2020. [publicação online] 2020 [cited 2020 Oct 25]. Available from: https://coronavirus. saude.gov.br/boletins-epidemiologicos.

11. Ministério da Saúde (Brasil). Boletim Epidemiológico- Centro de Operações de Emergência em Saúde Pública/Doença pelo Coronavírus 2019(COE-COVID19). Boletim 02 emitido em 02/04/2020. [publicação online], 2020 [cited 2020 Oct 25]. Available from: https://coronavirus.saude.gov.br/boletins-epidemiologicos.

12. Secretaria de Estado da Saúde (Maranhão). Boletim Epidemiológico COVID-19. Boletim atualizado em 29/10/2020. [publicação online] 2020 [cited 2020 Oct 29]. Available from: http:// www.saude.ma.gov.br/boletins-COVID-19/.

13. Zhu $\mathrm{Na}$, et al. A novel coronavirus from patients with pneumonia in China, 2019. New England Journal of Medicine [Internet]. 2020 Feb 20 [cited 2020 Oct 15]:727-733. DOI 10.1056 / NEJMoa2001017. Available from: https://www.nejm.org/doi/ pdf/10.1056/NEJMoa2001017?articleTools=true

14. Wang Chen, et al. A novel coronavirus outbreak of global health concern. The Lancet [Internet]. 2020 Jan 24 [cited 2020 Oct 15];395(10223):470-473. DOI https://doi.org/10.1016/ S0140-6736(20)30185-9. Available from: https://www.thelancet. com/journals/lancet/article/PIIS0140-6736(20)30185-9/fulltext?fbclid=IwARO_jIY1L9TutpZCO4PoKJsC8vNEnu5Vq_x582iyULg-9ML2UHF67Kw3_kE

15. Surveillances Vital. The epidemiological characteristics of an outbreak of 2019 novel coronavirus diseases (COVID-19) - China, 2020.ChinaCDCWeekly [Internet]. 2020 [cited20200ct15];2:113120. DOI 10.3760 / cma.j.issn.0254-6450.2020.02.003. Available from: https://pubmed.ncbi.nlm.nih.gov/32064853/.

16. Silva Amanda Priscila de Santana Cabral, Maia Lívia Teixeira de Souza, Souza Wayner Vieira de. Síndrome Respiratória Aguda Grave em Pernambuco: comparativo dos padrões antes e durante a pandemia de COVID-19. Ciência \& Saúde Coletiva [Internet]. 2020 [cited 2020 Sep 16];25:4141-4150. DOI https:// doi.org/10.1590/1413-812320202510.2.29452020. Available from: https://www.scielosp.org/article/csc/2020.v25suppl2/4141-4150/pt/. 